\title{
Systemic Therapy of Advanced/Metastatic Breast Cancer - Current Evidence and Future Concepts
}

\author{
Cornelia Liedtke $^{a}$ Hans-Christian Kolberg ${ }^{b}$ \\ a Department of Gynecology and Obstetrics, University Hospital Schleswig-Holstein/Campus Lübeck, Lübeck, Germany; \\ ${ }^{b}$ Department for Gynecology and Obstetrics, Marienhospital Bottrop, Bottrop, Germany
}

\section{Keywords}

Metastatic breast cancer - Molecular breast cancer subgroups - Chemotherapy - Targeted therapy ·

Endocrine therapy · Clinical trials

\section{Summary}

Systemic therapy of metastatic breast cancer may include chemotherapy, endocrine therapy, and targeted therapies (e.g., antibody-based approaches). These agents may be employed alone or in combination based on the patient's breast cancer subtype. Therefore, characterization of the disease phenotype is necessary and may include biopsy of the metastatic site. Novel therapeutic approaches include immunologic therapies, PARP inhibitors, PI3K inhibitors, and CDK4/6 inhibitors, which are currently under investigation in clinical trials.

(c) 2016 S. Karger GmbH, Freiburg

\section{Introduction}

\section{Breast Cancer - a Heterogeneous Entity}

It is well recognized that breast cancer is composed of several molecular subtypes that show marked differences with regard to both tumor biology and clinical behavior. At present, breast cancer is understood to comprise 3 major subtypes of clinical relevance:

Luminal breast cancer is defined by the presence of hormone receptor (HR) expression. It is commonly subdivided into luminal A breast cancer (which is usually highly endocrine-sensitive and slowly proliferating, resulting in a favorable prognosis) and luminal B breast cancer (which is less endocrine-sensitive and shows a higher proliferation rate, resulting in a less favorable prognosis).

Human epidermal growth factor 2 (HER2)-positive breast cancer is defined by an overexpression/amplification of HER2/neu. This is regarded as a prerequisite for an increased chance of response to novel HER2-targeted agents including trastuzumab, pertuzumab, and lapatinib. Importantly, HER2-positive/HR-positive breast cancer and HER2-positive/HR-negative breast cancer show significant differences both with regard to disease biology and clinical behavior.

Triple negative breast cancer (TNBC) is characterized by a lack of $\mathrm{HR}$ expression as well as a lack of overexpression/amplification of HER2/neu. As a consequence, neither endocrine treatment nor HER2-targeted agents are indicated. Instead, chemotherapy represents the most important treatment choice in all disease settings. Overall, this breast cancer subtype shows an unfavorable prognosis with high rates of recurrence and rapid progression in advanced disease stages. However, the prognosis of patients with TNBC is heterogeneous and highly dependent on their response to chemotherapy: In the case of response to chemotherapy, the prognosis may be very favorable [1].

\section{Molecular Heterogeneity of Metastatic Breast Cancer}

There is a solid body of scientific evidence showing that both HR expression and HER2/neu status may vary during the development of metastatic disease. Overall discordance rates between primary tumors and metastatic disease for estrogen receptor (ER), progesterone receptor (PR), and HER2 status have been reported to be as high as $20 \%$ (95\% confidence interval (CI) 16-35\%), 33\% (95\% CI 29 $38 \%$ ), and $8 \%$ (95\% CI 6-10\%), respectively [2]. Consequently, biomarkers that are associated with prognosis in the primary disease setting may not predict the outcome of metastatic disease. For in-

\section{KARGER}

(c) 2016 S. Karger GmbH, Freiburg 
stance, in their clinical study, Jeselsohn et al. [3] stratified patients with HR-positive metastatic breast cancer randomized to fulvestrant into luminal A and B breast cancer and could not demonstrate significant prognostic differences between the 2 molecular subgroups.

It is important to recognize, however, that the biological process that leads to receptor discordance is unknown in many cases and in fact may result from several biological and technical phenomena. These include tumor heterogeneity, changes in receptor status as a result of (targeted) treatment, technical issues (fixation schedules, decalcification protocols), and influence of the tumor microenvironment. Nevertheless, it is encouraged by current guidelines that a metastatic site is biopsied in order to i) verify the breast cancer molecular phenotype of the given disease stage, and ii) allow for a well-tailored treatment approach. However, it has to be recognized that data allowing an evidence-based approach in the case of receptor alterations is limited. Hence, there may be uncertainty as to how to react to a 'change in receptor expression' in a clinical setting. While there may be consensus regarding the initiation of a targeted therapy based on 'de-novo' expression of a target (such as endocrine therapy in a patient whose early breast cancer was HRnegative but now presents with HR-positive metastatic breast cancer), there are other scenarios which represent a challenge to the treating physician:

\section{Heterogeneity within a Patient}

Given that breast cancer (and metastatic breast cancer in particular) is well known to be highly heterogeneous, metastatic sites in a given patient may very well represent distinct molecular entities and therefore respond differentially to a certain therapy. Therefore, the optimal number and sites of biopsies are not defined and in a clinical setting may not be achieved.

\section{Loss of Therapeutic Target}

Both for HR expression and overexpression/amplification of HER2, changes from 'positive' to 'negative' have been described. However, due to the paucity of data, there is yet no evidence-based recommendation as to how to react to a 'loss' of a given therapeutic target, particularly if endocrine therapy is considered as a maintenance option after induction chemotherapy. Continuation of therapy may be considered in the case of potential disease heterogeneity by some but may be rejected by others based on concerns ('primum nihil nocere').

\section{Minimal Residual Disease}

Physicians that consider following the disease phenotype in a patient with metastatic breast cancer may face the necessity of repeat invasive procedures. One possible solution for this dilemma could be the detection and subtyping of circulating tumor cells in the peripheral blood (often referred to as liquid biopsy). This may facilitate i) the diagnosis of discordance at the very beginning and before it can translate into a metastasis with features different from the primary or other metastases, and ii) a targeted therapy to be designed accordingly. The DETECT study program is currently investigating this approach [4].

\section{Endocrine Therapy}

\section{Current Treatment Modalities}

In HR-positive metastatic breast cancer, endocrine therapy is the therapy of choice [5]. Only in cases of acutely life-threatening disease progression chemotherapy should be chosen in ER-positive/HER2-negative disease. If no such indication exists, endocrine therapy should be the preferred choice.

In the 1970s, tamoxifen was established as the therapy of choice for metastatic breast cancer with response rates between 16 and $56 \%$ and a superior toxicity profile compared to the former standard (high-dose estrogen) [6-13]. Although the median time to progression with tamoxifen is only about 6 months, the response is robust with many patients responding for 12-18 months, in rare cases even years. Therefore, tamoxifen is still a valid option in the endocrine therapy of metastatic breast cancer but has over the years been replaced by the aromatase inhibitors in postmenopausal patients as the treatment of choice in the first line. Response rates and time to progression of aromatase inhibitors are superior to tamoxifen whereas their toxicity profile is comparable [14].

Results concerning a comparison between tamoxifen and anastrozole are contradictory: in one study, no significant superiority to tamoxifen could be seen [15] whereas another investigation published at the same time showed a better progression-free survival (PFS) and clinical benefit rate [16]. In contrast, letrozole and exemestane proved to have a significantly better effect on response rate, clinical benefit rate, and PFS [17-19].

Although there are competitors on the horizon - as will be discussed below - and overall survival (OS) benefits compared to the old standard tamoxifen could not be shown in the trials mentioned above, aromatase inhibitors still represent a mainstay in the endocrine therapy of metastatic breast cancer in postmenopausal women.

Another option in the endocrine therapy of metastatic breast cancer is the selective ER downregulator fulvestrant. It completely lacks the partial estrogen agonism that i) may be a reason for treatment failures, and ii) certainly is the reason for the elevated endometrial cancer risk associated with tamoxifen [20]. Whereas first comparisons of fulvestrant with tamoxifen and aromatase inhibitors using a dose of $250 \mathrm{mg}$ showed no significant benefit [21-23], the CONFIRM trial comparing 250 to $500 \mathrm{mg}$ found that the latter dose is significantly superior [24]. Recent data showed superiority of fulvestrant $500 \mathrm{mg}$ to anastrozole in the first-line setting in a phase II study [25]. The ongoing phase III trial FALCON comparing fulvestrant and anastrozole in the first line will hopefully answer the question of where in the endocrine treatment cascade of metastatic breast cancer fulvestrant will find its place.

Most of the data above apply only to postmenopausal patients. It is self-explanatory that the principle of aromatase inhibition only works in patients with cessation of sex hormone production. However, treatment with tamoxifen may also present challenges. One of the main caveats of tamoxifen in premenopausal patients with intact ovaries is its ability to cause high peaks of estrogen 
with unknown and possibly damaging effects on the course of the disease. Ovarian suppression by luteinizing hormone agonists provides a solution for this dilemma. A large meta-analysis showed significant benefits in terms of response rates, PFS, and OS [26] for the combination of luteinizing hormone agonists and tamoxifen, and established this therapy as the standard for premenopausal patients.

\section{Future Therapies}

\section{PI3K/mTOR Inhibitors}

The phosphatidylinositol-3-kinase (PI3K)/protein kinase $\mathrm{B}$ (AKT)/mammalian target of rapamycin (mTOR) pathway plays an important role in cell growth, survival, and proliferation, and represents a central part of signal transduction in the cellular metabolism [27]. The pathway is activated by growth factors leading to phosphorylation of PI3K. One of the inhibitors of the PI3K/AKT/ mTOR pathway is phosphatase and tensin homolog (PTEN), a tumor suppressor that inhibits the activation of AKT. As in many other tumors, this pathway is activated in breast cancer. This happens either because of mutations in PTEN, PIK3CA, or AKT or because of amplification/mutation of receptor tyrosine kinases such as HER2. Since there is intensive crosstalk between the signaling of the ER and $\mathrm{PI} 3 \mathrm{~K}$, the $\mathrm{PI} 3 \mathrm{~K} / \mathrm{AKT} / \mathrm{mTOR}$ pathway is considered to play a major role in the development of endocrine resistance [28]. However, the prognostic relevance of mutations in the genes encoding for PI3K for HR-positive/HER2-negative breast cancer is limited. Although there was positive correlation between HR expression and PIK3CA mutations, no impact on OS could be shown in a recent meta-analysis [29].

Nevertheless, the inhibition of this pathway may represent a way of overcoming endocrine resistance in combination with fulvestrant or aromatase inhibitors. Supplementary table 1 (www.karger.com/?DOI=447549) summarizes the results of the most important studies on PI3K and mTOR inhibition in hormone-sensitive metastatic breast cancer. Whereas therapies targeting PI3K are still in (clinical) development, the combination of the MTOR inhibitor everolimus with the steroidal aromatase inhibitor exemestane is already a standard therapy after failure of a non-steroidal aromatase inhibitor. If the patient has not received a non-steroidal aromatase inhibitor in the adjuvant setting, therapy with everolimus and exemestane may be used in second or higher lines, whereas patients pretreated with anastrozole or letrozole may receive the combination of an mTOR inhibitor and a steroidal aromatase inhibitor as a first-line therapy option.

\section{CDK4/6 Inhibitors}

The family of cyclin-dependent kinases (CDK) is an important factor in the regulation of the cell cycle. CDK4 und 6 and cyclin D are responsible for transition from the $\mathrm{G} 1$ phase to the $\mathrm{S}$ phase through phosphorylation of retinoblastoma $(\mathrm{pRb})$ and thus are crucial for the (malignant) cell cycle [30]. Inhibitors of CDK4/6 ac- tivity such as palbociclib, ribociclib (LEE011), and abemaciclib are currently undergoing intensive development in metastatic breast cancer.

In 2009, the inhibitory effect of palbociclib on ER-positive breast cancer cells was demonstrated in vitro [31]. This fueled the further development of the substance in the setting of advanced ER-positive breast cancer. A phase II study (PALOMA-1) comparing letrozole plus palbociclib in the first line of therapy for metastatic or advanced disease resulted in a prolongation of PFS from 10.2 to 20.2 months and led to an accelerated approval of palbociclib in combination with letrozole as first-line endocrine therapy by the FDA in the USA. A phase III trial (PALOMA-2) has recently completed accrual [32]. For patients after failure of endocrine therapy, a phase III clinical trial (PALOMA-3) demonstrated a median PFS of 9.2 months with palbociclib and fulvestrant compared to 3.8 months with fulvestrant alone [33]. After these promising results, approval in Europe is expected in combination with either letrozole or fulvestrant.

The available data for abemaciclib and ribociclib (LEE011) also show clinical efficacy and no unexpected warning signs [34, 35]. Supplementary table 2 ( $w w w$. karger.com/?DOI=447549) presents the current study portfolio for CDK4/6 inhibition in metastatic breast cancer.

In summary, CDK4/6 inhibitors are one of the most promising innovations in the management of breast cancer in recent years and are expected to change the course of endocrine therapy.

\section{Choice and Sequencing of Endocrine Therapies}

Overcoming endocrine resistance or preventing it before it can develop is a paradigm shift in endocrine therapy of metastatic breast cancer. Patients who used to be candidates for chemotherapy may continue endocrine therapy in combination with new agents aiming at overcoming endocrine resistance. Even more promising are data for the use of these agents in first-line endocrine therapy of metastatic breast cancer, thereby preventing development of endocrine resistance. This will reduce the use of chemotherapy in endocrine-responsive patients to an extent not seen before. However, this is not the only aspect that will change. In fact, the increasing number of endocrine treatment options calls into question the concept of treatment choices being made according to line of therapy and rather encourages treatment tailoring according to comorbidities and preferences of the patient. Several agents targeting PI3CK, CDK4/6, and mTOR will soon become available, and the choice thereof will depend on the experience of the physician as well as patient-related factors mentioned above. New toxicities previously unknown to endocrine therapy such as stomatitis for mTOR inhibitors or mental disorders for PI3K inhibitors have to be taken into account, and physicians will have to learn how to handle them.

Only few studies are investigating the use of a specific new agent after failure of another specific new agent, e.g. a PI3K inhibitor after failure of e.g. an mTOR inhibitor (BELLE-3 study) [36]. The 
new landscape will change how trials are designed, away from investigating the efficacy of a study medication after failure of a specific therapy and towards options with broader inclusion criteria. This will certainly be necessary for the sake of recruitment, and also - and this will be beneficial for real life - for providing evidence not limited to a small number of patients.

The new wealth of options will make it easier to tailor therapies according to the needs of the individual patient; however, it will also make it harder to decide what is the right therapy at the right moment. The era of algorithms is over.

\section{Chemotherapy for Metastatic Breast Cancer}

\section{Indication for Chemotherapy in Metastatic Breast Cancer}

The most important goal in the metastatic setting is the preservation/improvement of quality of life. Hence, chemotherapy is indicated in limited situations only. An indication for chemotherapy may be seen in the following scenarios:

Patients with metastatic HR-positive/HER2-negative breast cancer unsuitable for endocrine intervention alone due to i) (recurrent) resistance to endocrine interventions or ii) rapid (and therefore potentially life-threatening) disease progression resulting in a high need for disease remission;

Patients with metastatic TNBC requiring chemotherapy, given that as of yet there are no relevant targets suitable for targeted therapy outside of clinical trials;

Patients with metastatic HER2-positive breast cancer due to the necessity of chemotherapy as part of the majority of HER2-targeted treatment regimens.

\section{Mono- versus Polychemotherapy}

In cases with an indication for chemotherapy, monochemotherapy is usually applied. Polychemotherapy compared to monochemotherapy may increase response rates and even prolong PFS, at the cost however of an increased rate of (potentially severe) treatment-associated toxicities. Therefore, combination chemotherapy should be restricted to patients with severe symptoms and a high need for remission. In those rare cases, the combination of taxanes with either anthracyclines or antimetabolites represents a suitable regimen.

For monotherapy, a large number of agents may be considered: taxanes (paclitaxel/docetaxel/nab-paclitaxel), anthracyclines (epirubicin/doxorubicin/(pegylated) liposomal doxorubicin), platinum compounds (carboplatin/cisplatin), vinorelbine, capecitabine, eribulin, and gemcitabine.

Although the optimal duration of chemotherapy repeatedly represents a matter of debate, there is solid consensus that the duration of a given chemotherapy regimen should be restricted to the patient's therapeutic index (i.e., therapeutic efficacy vs. therapeutic toxicity) remaining positive [37].

\section{Choice of Chemotherapy Agents}

In some cases (such as in HER2-targeted therapy), the choice of certain chemotherapy agents may be encouraged by the fact that efficacy has been demonstrated in certain combination regimens only. Examples of such may be the choice of docetaxel with pertuzumab/trastuzumab or capecitabine with lapatinib. In other cases, the selection of chemotherapy agents is less strict. Since taxanes and anthracyclines are considered to represent the most efficacious agents in breast cancer chemotherapy, they should be used before other agents are selected unless there are contraindications or these agents have already been used in earlier disease stages. Other than that, there are several aspects that may guide the choice of chemotherapy in the clinical setting [38]: ER/PR, HER2 status; indication for combination with biologicals; previous treatments (and their toxicities); recurrence-free interval following adjuvant therapy; aggressiveness of disease, site metastasis; estimated survival time; concurrent diseases (including organ function); and expectations/ preference of the patient.

These recommendations strongly emphasize patient-associated parameters when deciding about a specific cytostatic agent. In contrast, there is yet limited but increasing scientific evidence as to whether certain breast cancer molecular subtypes (such as TNBC) derive particular benefit from specific chemotherapy agents (such as eribulin) [39]. Although these data are encouraging, physicians should be cautious to base their choice of chemotherapy agents solely upon subgroup analyses. Instead, the above criteria should be employed when choosing a certain chemotherapy agent.

\section{Choice of Chemotherapy for Patients with Metastatic TNBC}

There is an increasing body of scientific evidence supporting a particular role of platinum salts in the palliative therapy of patients with hereditary breast cancer (i.e., those carrying a $B R C A 1 / 2$ mutation). For a long time, evidence was limited to data from retrospective analyses [31]. This, however, has changed following the publication of the results from the British TNT (Triple Negative Trial) study which randomized patients with either metastatic TNBC or hereditary (i.e., BRCA1/2-associated) breast cancer to i) taxane monotherapy (docetaxel $100 \mathrm{mg} / \mathrm{m}^{2}$ every 3 weeks ( $\mathrm{q} 3 \mathrm{w}), 6$ cycles) or ii) carboplatin (area under the curve $6, \mathrm{q} 3 \mathrm{w}, 6$ cycles) [40]. Response rates following 3-6 cycles of chemotherapy showed a significant difference in favor of platinum-based therapy among patients with hereditary breast cancer: response rates for carboplatin versus docetaxel were 68 versus 33\%, respectively $(\mathrm{p}=0.03)$. Among patients with metastatic non-hereditary breast cancer, no significant association was observed (28 vs. $37 \%$; $\mathrm{p}=$ 0.16). In Germany, the AGO (Arbeitsgemeinschaft Gynaekologische Onkologie, Gynecologic Oncology Working Group) prefers the use of platinum salts (i.e., carboplatin) to taxanes (i.e., docetaxel) in patients with metastatic breast cancer carrying a $B R C A 1 / 2$ mutation. 


\section{Targeted Agents for Metastatic Breast Cancer}

Antiangiogenic Therapy in Patients with Metastatic Breast Cancer acknowledged that antiangiogenic therapies - while under intense development for other malignancies such as ovarian cancer [49] are not living up to their promise in breast cancer.

\section{HER2-Positive Breast Cancer - How to Choose the Optimal HER2-Targeted Agent?}

HER2-positive breast cancer has undergone a paradigm change from a highly aggressive subtype with a particularly unfavorable prognosis to a subtype that is considered as being the best treatable entity due to the HER2 receptor representing an optimal target for antibody-mediated therapy: in fact, the development of the monoclonal HER2-targeted antibody trastuzumab has changed our perception of HER2-positive breast cancer dramatically [50] - despite usually representing a fast proliferating, highly aggressive entity, HER2-positive disease is currently regarded as one of the subtypes with the largest number of highly efficacious treatment options.

Trastuzumab is a humanized monoclonal antibody targeted against the extracellular domain of the HER2 receptor and has been shown to significantly improve OS in combination with monochemotherapy [51]. In addition to trastuzumab, pertuzumab is a monoclonal antibody directed against the HER2/HER3 dimerization domain of the HER2 receptor, which has just recently been approved for the first-line treatment of HER2-positive breast cancer in combination with docetaxel and trastuzumab. Patients treated with this combination chemotherapy may reach OS rates of as high as 56.5 months [52]. Consequently, this regimen is commonly considered today's standard first-line chemotherapy regimen for patients with HER2-positive metastatic breast cancer.

Trastuzumab emtansine (TDM-1) is a highly effective antibodydrug conjugate (ADC) of the cytotoxic DM-1 and trastuzumab. After internalization of the ADC, the cytotoxic agent is released and may target the tumor cell from the inside. In the pivotal EMILIA study, use of TDM-1 was demonstrated to significantly increase both PFS and OS rates compared to capecitabine plus lapatinib (OS 30.9 vs. 25.1 months, respectively) [53]. Consequently, TDM-1 was approved for use in patients with HER2-positive metastatic breast cancer either as second-line treatment regimen or as first-line option in the case of fast progression ( $<6$ months) after a trastuzumab-based treatment regimen in the curative setting.

In addition to HER2-directed antibodies, HER2 signaling can be abrogated by the use of small HER2-directed molecules. Among those, lapatinib (targeting both HER1/EGFR and HER2) was the first small molecule and second HER2-targeted agent that was registered for HER2-positive metastatic breast cancer in combination with capecitabine based on the results of a randomized study demonstrating a PFS benefit of approximately 4 months for the combination of capecitabine/lapatinib compared to capecitabine alone $(\mathrm{p}<0.001)$ [54]. The role of lapatinib, however, has lessened since the development of pertuzumab and T-DM1 since both agents have been demonstrated to yield an OS benefit. Therefore, lapatinib is largely recognized as a treatment option for later-line therapy of HER2-positive disease. 
Neratinib is a novel member of the family of HER2-targeted agents. The small molecule irreversibly targets HER1, HER2, and HER4, and is not yet licensed for the treatment of breast cancer [55]. The agent, however, demonstrated significant efficacy in an adjuvant study presented at the recent American Society of Clinical Oncology (ASCO) meeting 2015 [56] and is currently under investigation in several trials in the metastatic setting.

\section{Choice/Sequencing of HER2-Targeted Agents}

Given that several agents are listed and several more are underway, there is considerable doubt that sequencing of HER2-targeted agents will continue to be carried out as it is currently being done. When deciding about HER2-targeted agents in the metastatic setting, licensing issues are of high relevance and guide decisions as to what agent to employ in which line setting, given that licensing is yet strongly limited to certain treatment-lines. For instance, the antibody pertuzumab is licensed for the first-line setting, leaving patients with later-line therapies no in-label option for treatment with this highly effective antibody. The biologic background for using these agents in a strict order is limited. Furthermore, if we take into account the basic principles of choosing systemic therapy in the metastatic setting, physicians are led to putting less emphasis on factors such as patient choice or side effects. It seems plausible (and necessary) to eventually lower these limits when choosing the optimal treatment sequence for patients with metastatic HER2positive breast cancer.

\section{Promises of Novel HER2-Targeted Agents}

Besides overcoming disease resistance though optimized sequencing, one of the major promises of HER2-targeted agents is that they allow for i) therapy escalation through highly effective, optimal combination regimens; and ii) therapy de-escalation through replacement of chemotherapy with HER2-targeted agents.

Chemotherapy-free HER2-targeted treatment regimens for instance have already been licensed with regard to lapatinib therapy: lapatinib is registered for the treatment of HER2-positive/HR-positive breast cancer in combination with letrozole [57] and for patients with HER2-positive/HR-negative breast cancer in combination with trastuzumab [58]. Other regimens are currently under investigation.

\section{Defining and Predicting Resistance against HER2-Targeted Agents}

There is an increasingly solid body of scientific evidence suggesting that alterations in the PI3K/AKT/mTOR pathway may also mediate resistance against HER2-targeted agents among patients with HER2-positive breast cancer. Several analyses including 1 recent meta-analysis demonstrated that alterations in PI3K signaling such as PIK3CA alterations may confer resistance against dual blockade with trastuzumab and lapatinib resulting in decreased pathologic response rates in patients undergoing neoadjuvant therapy; furthermore, an association with adverse survival outcome particularly in HER2-positive/HR-positive subtypes has been suggested [59].
The BOLERO-1 and BOLERO-3 studies evaluated the value of mTOR inhibition through everolimus in patients with HER2-positive metastatic breast cancer in combination with trastuzumab. In a translational analysis based on patient samples derived from these studies, Slamon et al. [60] recently demonstrated an association between alterations in the PI3K/mTOR cascade and efficacy of everolimus in reversing trastuzumab resistance. These early analyses suggest that targeting of the PI3K/mTOR cascade may be employed not only to reverse endocrine resistance but also to reverse resistance against HER2-targeted agents in certain HER2-positive subtypes.

\section{$T N B C$}

Tumor Immunology as Targeted Therapy for Metastatic TNBC

It has been well demonstrated as part of numerous scientific analyses that tumor cell versus immune cell interaction seems to play an important role in breast cancer in general and TNBC in particular. In fact, patients with TNBC derive increased benefit from neoadjuvant chemotherapy if their tumor carries a strong lymphocytic infiltrate.

Consequently, immune-targeted agents are currently under intensive investigation in several malignant entities including (triple negative) breast cancer. These agents are understood to lead to a demasking of the malignant cell to the immune system. The efficacy of this type of agent is therefore thought to strongly depend on the mutational frequency of the tumor type. Therefore, some of the most promising results yet have been published in the context of malignant melanoma. However, both an increased mutational frequency and a pronounced need to develop novel therapeutic concepts other than chemotherapy have fostered the development of immune oncology also in the TNBC subtype. Both the programmed cell death protein 1 (PD-1) and its 2 ligands PD-L1 and PD-L2 are of particular interest as potential therapeutic targets in TNBC through humanized antibodies. In a phase I clinical trial, efficacy and safety of pembrolizumab (Keytruda ${ }^{\circledR}$, Merck, Kenilworth, NJ, USA) was analyzed among 27 patients with TNBC: 1 case of complete remission, 4 cases of partial remission, and 7 cases of stable disease were assessed. No worrisome safety signals were recorded [61].

\section{DNA Damage Repair as a Potential Therapeutic Target in} Metastatic TNBC

The enzyme poly-(ADP-ribose) polymerase (PARP) is involved in a large number of intracellular processes including repair of DNA damage. Inhibition of PARP in tumor cells may keep a tumor cell from repairing DNA damage that may result from either radiation or cytotoxic therapy [62]. BRCA1/2 mutations confer an impaired DNA damage capacity. Therefore, inhibition of DNA damage repair through iatrogenic PARP inhibition may represent an aspect of particular interest in patients with (metastatic) TNBC.

The mechanism of action of PARP inhibitors is often referred to as synthetic lethality: First, the combination of a genetic and a somatic mutation leads to the inhibition of BRCA function which is, 
secondly, followed by iatrogenic inhibition through application of PARP inhibitors which may abrogate the compensatory role of PARP. Several PARP inhibitors are currently under intensive investigation for all stages of triple negative and/or hereditary (i.e., $B R C A$-associated) breast cancer.

TNBC Subtypes and Targeted Therapeutic Agents

Rather than constituting a homogeneous entity, TNBC is increasingly understood as being quite heterogeneous. In fact, high-throughput genomic analyses of TNBC suggest that TNBC may be subdivided in at least 6 distinct molecular subtypes. The basal-like 1 TNBC subtype seems to be predominant in hereditary breast cancers and therefore may benefit from targeting of DNA damage repair through agents such as PARP inhibitors. In contrast, the immunomodulatory TNBC subtype shows an abundance of immune signals and therefore may represent a subgroup which immunomodulatory agents can target particularly well. One TNBC subtype that may be of particular interest in the context of novel treatment options is the luminal androgen receptor (AR)-positive subtype. Recent data from patients with metastatic AR-positive TNBC suggests that these patients may derive some benefit through treatment with antiandrogens [63].

These analyses demonstrate that among heterogeneous entities such as TNBC, molecular insight into certain disease subgroups is essential in order to best identify relevant mechanisms that represent targetable Achilles heels of each individual disease subtype. These mechanisms might not become evident if the disease were to be analyzed as a whole.
There is yet, however, no data demonstrating the value of TNBC substratification in the metastatic setting. Given that TNBC subtypes have been associated with distinct prognoses, the distribution of TNBC subtypes in metastatic disease may vary. Furthermore, it has not yet been analyzed whether molecular features of TNBC subtypes remain stable during the metastatic cascade or may be altered upon disease progression (and particularly in the face of targeted agents).

\section{Online Supplementary Material}

Supplementary Table 1. Results for the use of mTOR and PI3K inhibitors in hormone-sensitive metastatic breast cancer, modified from [64].

Supplementary Table 2. Active studies with CDK4/6 inhibitors in metastatic breast cancer.

To access the supplementary material, please refer to www.karger.com/? DOI $=447549$.

\section{Disclosure Statement}

The authors have the following potential conflicts of interest with respect to the content of this manuscript to declare: CL has received honoraria from Amgen, Celgene, Eisai, GSK, Novartis, Pierre-Fabre, Roche, and TEVA. HCK has received honoraria from Novartis, GSK, Pfizer, Roche, TEVA, Amgen, Janssen, and LIV Pharma.

\section{References}

References can be found online ( $w w w \cdot k a r g e r . c o m / ? D O I=447549$ ). 